\title{
Procesos semánticos en los textos filosóficos de Nietzsche y su función filosófica: Algunos resultados de la investigación sobre el Diccionario-Nietzsche ${ }^{1}$
}

\author{
GERD SCHANK, HERMAN SIEMENS, PAUL VAN TONGEREN \\ Universidad de Nijmegen (Holanda)
}

Queremos hacer en este artículo algunas observaciones sobre el tema del uso del lenguaje en Nietzsche y describirlo de una manera más precisa. Nos concentramos para ello sobre un número de observaciones contextuales de su lenguaje, que están unidas en muchas aspectos con su proyecto filosófico. El pensamiento de Nietzsche puede caracterizarse por el intento de hacer justicia a la multiplicidad y al cambio de una manera radical; una caracterización que lo sitúa no sólo en la oposición clásica, al lado de Heráclito y frente a Parménides, sino que lo distingue propiamente de aquello que puede ser considerado como la línea dominante en toda la historia de la Filosofía desde Platón. Muchos conceptos clave de su pensamiento remiten a esta caracterización central: la voluntad de poder y el papel de la lucha; el perspectivismo y el sentido de la interpretación; la genealogía y así sucesivamente: todos ellos tienen relación con el hecho de que en Nietzsche figura en un primer plano la utilización de los términos 'unidad' y 'Ser', 'multiplicidad' y 'devenir'.

De un pensador como Nietzsche seguramente se puede esperar que una tal idea fundamental se exprese también en su uso del lenguaje. Nietzsche no sólo era como filólogo un experto en cuestiones del lenguaje, era también como artista un creador del lenguaje y con el lenguaje, y como filósofo estaba convencido ante todo del influjo del lenguaje en el pensamiento y viceversa: también los esquemas mentales y las tradiciones intelectuales se suprimen permaneciendo en el lenguaje. De ahí que el interés por la multiplicidad y el cambio se reflejen no sólo en su lenguaje; el lenguaje mismo, según la concepción de Nietzsche, es al mismo tiempo también el producto de una realidad cambiante y polisémica, y es por encima de ello el instrumento, más aún, el arma para romper el poder de toda dogmatización, con lo cual el lenguaje es, sin embargo, con frecuencia un instrumento resistente, porque está encadenado a aquello que lo intenta romper.

\footnotetext{
${ }^{1}$ Nota del traductor: Publicamos este artículo en traducción castellana, pero es ésta su primera publicación tanto en castellano como en sus lenguas originales holandesa y alemana.
} 
En este artículo, sin embargo, no nos ocuparemos en un primer plano a una tal definición filosófica e interpretación del lenguaje de Nietzsche, sino que nos limitaremos principalmente a la descripción de unas cuantas de sus observaciones típicas ( $\$ 2$ ). Sólo al final formularemos algunas propuestas para una interpretación filosófica de las observaciones descritas (§3). Comenzamos con una introducción, que informa brevemente sobre la investigación de la que procede esta aportación $(\S 1)$

\section{NECESIDAD Y MÉTODO DE UNA INVESTIGACIÓN DEL USO DEL LENGUAJE EN NIETZSCHE}

Los filósofos se esfuerzan en general por conseguir definiciones y conceptos claros. Aspiran en su uso del lenguaje, en la medida de sus posibilidades, de una relación reciproca entre significante y significado, y ciertamente a que sea lo más independiente posible del contexto. En Nietzsche a penas o nada se puede hablar de eso, como lo pueden aclarar un par de manifestaciones procedentes de la investigación de Nietzsche. Según la apreciación de Mette, Nietzsche es un «descubridor de palabras y un acuñador de expresiones kat'exochēn»'. Según la concepción de Müller-Lauter, Nietzsche nombra «con el mismo concepto sus peculiaridades valoradas de manera distinta $»^{2}$. Ansell-Pearson habla de la 'polisemia' de muchas palabras claves de Nietzsche y pone como ejemplo el término 'superhombre'. Más adelante él cree poder dar cuenta de un ejemplo de Nietzsche con las 'connotaciones' de la preposición über (sobre), por ejemplo Über-mensch (super-hombre) ${ }^{3}$. Willers pone de relieve que en el Zaratustra «no pocas palabras tienen nuevas connotaciones ${ }^{4}$. Politycki habla incluso de la «semántica privada» de Nietzsche ${ }^{5}$, de «la praxis lingüística parecida a un laberinto» de Nietzsche ${ }^{6}$, de una «traducción del término individual» ${ }^{7}$, así como «los cambios de sentido de Nietzsche unívocos, dobles o múltiples» ${ }^{8}$. Él habla además del «uso estrictamente dialéctico de la palabra» en Nietzsche (en la

${ }^{1}$ A. Mette, Zur Psychologie des Dionysischen. In: Imago. Zeitschrift für Anwendung der Psychoanalyse auf die Geisteswissenschaften. Hg. Sigmund Freud. Leipzig, Wien u. Zürich. Nr. 20, 1934, 191-218. Aquí p. 216. (Citado en: M. Politycki, Umwertung aller Werte?, De Gruyter, Berlin, 1989, p. 105).

${ }^{2}$ W. Müller-Lauter, Nietzsche. Seine Philosophie der Gegensätze und die Gegensätze seiner Philosophie. Walter de Gruyter, Berlin, 1971, p. 1.

${ }^{3}$ K. Ansell-Pearson, Nietzsche contra Rousseau, Cambridge University Press, Cambridge, 1991, p. 159.

${ }^{4}$ U. Willers, Nietzsches antichristliche Christologie, Tyrolia, Innsbruck-Wien, 1988, p. 171.

${ }_{6}^{5}$ Politycki 1989, pp. 101 y 105s.

${ }^{6}$ Ibid., p. 107.

${ }^{7}$ Ibid., p. 109.

${ }^{8}$ Ibid., p. 106. Umbegreifung es una forma de analogía de Polityckis para la 'transvaloración' (Umwertung). 
polarización de los significados de una palabra), así como «del uso perspectivista múltiple de la palabra, cuando una palabra (significante) mantiene significados distintos según la perspectiva» ${ }^{9}$. El mismo Nietzsche era completamente consciente de ello: «Muchas palabras se han incrustado en mi con otras sales y tienen un sabor distinto tanto a mi paladar como para mis lectores ${ }^{10}$. También Peter Gast había observado esto, tal y como se puede ver, cuando escribe que «cada palabra de Nietzsche [tiene] un sentido propio, más profundo y penetrante que en los filósofos precedentes» ${ }^{11}$.

Estas características del uso del lenguaje de Nietzsche están llamativamente en contra de la accesibilidad relativamente grande de sus escritos, al menos aparentemente. Nietzsche pertenece a ese pequeño grupo de pensadores que son también muy leídos fuera del círculo de la filosofía especializada. Sus escritos no pertenecen sólo a la historia (y quizás incluso al canon) de la filosofía, sino que también pertenecen a la historia de la lengua y literatura alemanas. Este carácter accesible de sus escritos es, sin embargo, traicionero. No solamente se pasa por alto fácilmente el experimento filosófico que tiene lugar en sus escritos, sino que además se olvida con facilidad, que el alemán del siglo XIX de Nietzsche no es el alemán de hoy -aun dejando completamente a parte la circunstancia de que Nietzsche de vez en cuando recurre a significados antiguos de las palabras como a aquellos que en su época eran usuales ${ }^{12}$. Todavía hay que añadir que el lenguaje de Nietzsche, de manera más profunda que en muchos otros autores, ha sido infectado por la historia de la recepción. $\mathrm{Al}$ menos algunas expresiones de su lenguaje parecen haberse establecido en una interpretación dudosa tanto política como filosóficamente.

Por lo tanto, hay motivos más que suficientes para una investigación a fondo del uso del lenguaje en Nietzsche, y para un diccionario de Nietzsche, como el que está siendo preparando desde hace algunos años por un grupo de investigación internacional, que tiene su centro en Nimega (Holanda). En el último número de Estudios Nietzsche (3, 2003, 207-213) fue presentado ya brevemente este proyecto. Como anticipo a la aparición del primer tomo del Diccionario de Nietzsche, que saldrá como muy tarde este año, se presentan en este artículo algunos resultados de esta investigación.

El Diccionario de Nietzsche procede ante todo semasiológicamente, pero une este principio metodológico con elementos onomasiológicos. Eso significa que

${ }^{9}$ Politycki 1989, p. 112.

${ }^{10}$ Carta de Nietzsche a Brandes, 2-12-1887, KSB VIII 206.

${ }^{11}$ P. Gast, Observación previa a «Así habló Zaratustra», p. 2, citado en Politycki, op. cit., p. 109.

${ }^{12}$ Por ejemplo gemein (común), que Nietzsche también utiliza con el significado antiguo documentado en Lutero; o 'descubrir' (entdecken), que en él de vez en cundo tiene el significado antiguo de 'desenmascarar' (entlarven); o 'artificial' (künstlich), que a veces tiene todavía el significado de 'artificioso' (kusntvoll). También el término que ocupa un lugar central 'gegen' (contra) tiene en Nietzsche todavía un espectro de significado más amplio que el actual. Véase G. Schank, Dionysos gegen den Gekreuzigten, P. Lang, Bern, 1993. 
en primer lugar no está el concepto, sino el significante. De ese modo, pueden ser comprendidos mejor los cambios de significados, los nuevos significados así como la combinación de significados y connotaciones. También puede ser definido mejor el lugar histórico lingüístico del uso que hace Nietzsche de una palabra determinada. Además de eso, la palabra se sitúa sin embargo también en el (correspondiente) campo semántico, a través del cual son posibles otros exámenes adicionales en el uso del lenguaje de Nietzsche. De esta manera, se pueden poner de relieve cambios en el uso del significante individual a lo largo de la cronología de los escritos de Nietzsche, pero también cambios semánticos, y en tercer lugar, cambios en los puntos de referencia de Nietzsche respecto a estos conceptos. En esta reconstrucción del campo semántico, en el que una palabra mantiene su(s) significado(s), se lleva a cabo un planteamiento onomasiológico. Éste adquiere especial relevancia, cuando la utilización de Nietzsche de la palabra en cuestión es definida también histórico-conceptualmente, y cuando en la discusión con la investigación de Nietzsche en la literatura secundaria se describe el significado del concepto en el pensamiento de Nietzsche. En el presente artículo nos limitamos a la descripción de algunos resultados de la parte (primera) semasiológica del artículo en el Diccionario de Nietzsche ${ }^{13}$.

En él, la tarea principal es la recopilación y el poner en orden en la medida de lo posible los distintos significados, aspectos del significado y de los usos relevantes significativos de la palabra en cuestión. Para ese objetivo se ha investigado y consultado cada cita en la que aparece una palabra, entrando en detalles respecto al significado que tiene aquí la palabra. Esto conduce a una estructura compleja, en la que se distinguen a menudo una docena de distintos aspectos significativos. Aunque para cada una de las variantes distintas tomamos siempre como ejemplo una o más citas, la mayoría de las veces es imposible reducir a un único significado el uso de una palabra en un determinado texto. Los aspectos distintos y diferentes del significado suenan siempre todos simultáneamente, de una u otra manera, en el uso de Nietzsche de la palabra en cuestión. Sin embargo, es importante distinguir los significados diversos y los aspectos significativos. Categorías palmarias, que pueden ser usadas en esto, son por ejemplo: la distinción entre la interpretación que se mantiene en el significado usual de una palabra, frente a la interpretación propia de Nietzsche de esa palabra -por ejemplo, 'moral'

${ }^{13}$ Cuando a continuación se habla de 'palabra' (Word), se quiere expresar en primer término el 'significante'. Cuando luego se habla de 'procesos semánticos', se quiere decir con ello el hecho de que Nietzsche atribuye a una palabra uno ( o varios) significados. Cuando se habla de un 'nuevo' significado, aquí significa: un significado que hasta ahora no se ha unido todavía con la palabra. Las innovaciones lingüísticas de Nietzsche tienen lugar primeramente en el campo semántico. Dejando a parte que en el presente artículo el planteamiento onomasiológico no se va a seguir discutiendo, también se excluyen aquí aspectos pragmáticos y preformativos del uso del lenguaje de Nietzsche. Por lo tanto, se trata en lo que sigue de un proceso semántico en el sentido indicado. Nosotros hacemos siempre referencia en el presente artículo al trabajo ya elaborado para el Diccionario de Nietzsche, sin remitir explícitamente a él en cada ocasión. 
(Moral)-, o entre un significado metafísico o no metafísico de una palabra -por ejemplo, 'reflejo' (Abglanz)-; o entre una connotación neutral, una negativa y una positiva -por ejemplo, 'abreviatura' (Abbreviatur)-; o también las distintas clases de realidad (cosas, animales, hombres, comunidades), en cuyo contexto Nietzsche utiliza la palabra; etc. Nosotros elaboraremos un poco más adelante algunos criterios periódicos del uso polisémico del lenguaje en Nietzsche, que se ponen en claro a través de ese uso.

\section{Algunos PROCESOS SEMÁNTICOS CENTRALES EN LOS TEXTOS FILOSÓFICOS DE NIETZSCHE}

\subsection{CONNOTACIÓN Y CAMBIO DE CONNOTACIÓN}

Por connotación se entiende en lingüística las «ideas emocionales concomitantes de una palabra ${ }^{14}$. En el uso del lenguaje de Nietzsche tenemos una connotación positiva o negativa claramente reconocible para casi todas las palabras más importantes para su pensamiento. Generalmente son connotadas positivamente, por ejemplo, los griegos, la vida, lucha y guerra. Por el contrario, moral, rebaño y Platón lo son la mayoría de las veces negativamente.

Pero también hay en Nietzsche palabras cuya connotación cambia: la 'metáfora de las cadenas' aparece con una connotación positiva en el giro «bailar encadenado» (in Ketten tanzen) (MA IIb, §140, KSA II 612), pero la connotación es negativa si se habla de la «la enfermedad de las cadenas» (Ketten-krankheit), de la que los hombres deben ser curados (MA IIb 350, KSA II 702). En el mismo escrito, El caminante y su sombra, (MA IIb) una metáfora puede, por lo tanto, ser negativa o positiva según el contexto. La consideración de las connotaciones, y el cambio de connotación de modo especial, es particularmente importante en ciertas palabras explosivas, como por ejemplo, 'rebaño' (Herde) y 'degeneración' (Entartung), que en general tienen un valor connotativo exclusivamente negativo. También en Nietzsche la connotación negativa de 'degeneración' está documentada, cuando por ejemplo habla de la «degeneración del dominador» (KSA XI 102, 25[344]), con lo que él piensa en un desvío de la 'forma' en que se ha utilizado hasta ahora y especialmente en un dominador aristocrático. Además él usa sin embargo también la palabra 'degeneración' con una connotación positiva y en un contexto, que la concepción usual unida a esta palabra de una desvalorización y un movimiento descendente se invierte completamente, cuando él habla de un «ennoblecimiento por degeneración» (MA I §224, KSA II 187). Un tal cambio de connotación (y de significado) no pude ser pasado por alto. 'Degeneración' es utilizada en Nietzsche de diferente modo, y la utilización de esta palabra más recientemente,

${ }^{14}$ W. Ulrich, Wörterbuch Linguistische Grundbegriffe, Ferdinand Hirt., Kiel, 1972, p. 63. 
connotada exclusivamente de manera negativa, indicando una desvaloración, es decir, una depreciación, no puede por eso apoyarse en Nietzsche.

El cambio de connotación puede también estar unido al cambio de significado. Las palabras 'hombre culto' (Gebildete) y ‘formado' (gebildet) las utiliza siempre Nietzsche, cuando él comprende 'gebildet' en sentido tradicional, con una connotación negativa. Sin embargo, él usa la palabra con una connotación positiva, cuando piensa gebildet en su sentido, así por ejemplo cuando escribe: «Nuestra sociedad representa sólo la cultura [Bildung], falta el 'hombre culto' [Gebildete]» (KSA XII 220, 5[81]). El Nietzsche tardío concibe al 'hombre culto' como alguien que está formado (gebildet) de una 'manera orgánico-fisiológica'.

Las connotaciones son dadas a entender en Nietzsche la mayoría de las veces mediante el contexto correspondiente, pero en ocasiones también mediante comillas (así en el ejemplo de Gebildete), en donde además se dan la mano el cambio de connotación y el cambio de significado. Especialmente complicado resulta, por ejemplo, a este respecto con la palabra 'aventura' (Abenteuer). Aquí se pueden demostrar en Nietzsche dos significados, una aventura 'interna' y una aventura 'externa', que pueden ser connotadas las dos en un sentido positivo y negativo.

\subsection{CAMBio de SIGNIFICADO: ATRIbUCIÓN DE UN NUEVO SIGNIFICADO}

Debe hablarse de un cambio de significado, cuando Nietzsche sustituye el significado tradicional de una palabra por un significado nuevo, introducido por él de nuevo, y cuando él utiliza luego (más o menos) exclusivamente este nuevo significado en sus escritos siguientes. Como ejemplo podemos señalar: la palabra 'conciencia' (Bewusstsein), con la que Nietzsche define nuevamente y fundamenta conciencia sobre la base del inconsciente (instinto y sensaciones), $o$ también 'pensamiento' (Denken), que en él, a diferencia de la tradición filosófica, tiene su punto de partida en el pensamiento inconsciente del cuerpo ${ }^{15}$.

Si se plante la difícil cuestión de la relación entre conceptos tradicionales y los acuñados nuevamente por Nietzsche, entonces se podría quizás hablar aquí a primera vista de una divergencia. Sin embargo, la cuestión es más complicada. Mientras que, por ejemplo, el concepto de conciencia de Descartes se basa en el par de contrarios res extensa y res cogitans, en Nietzsche esta oposición se supera, en la medida en que él admite un tránsito flexible, una gradación del cuerpo, sus instintos y sensaciones hacia una conciencia y un pensamiento consciente que se construyen sobre ellos. Nietzsche deja a un lado el fundamento del concepto de conciencia de Descartes. Sus conceptos respectivos de conciencia parten de premisas completamente distintas y se encuentran, por decirlo así, sobre planos totalmente distintos, y son por eso también difícilmente comparables. Si se quiere hablar aquí todavía de una 'oposición' de los dos

${ }^{15}$ Más ejemplos en Politycki, op. cit., p. 105. 
conceptos de conciencia, entonces no se prestaría atención los fundamentos totalmente distintos, sobre los que descansan estos dos conceptos. Por lo tanto, no se hace justicia a la filosofía de Nietzsche y a sus innovaciones con esa manera de considerarlas solamente de modo puntual, puesto que pone entre paréntesis el contexto filosófico, en cuyo marco Nietzsche efectúa sus nuevas definiciones semánticas y filosóficas. Pues la atribución de nuevos significados no está nunca limitada en Nietzsche a palabras individuales. Nunca tiene lugar solo puntualmente, sino más bien la mayoría de las veces en el marco de sucesivas innovaciones, de tal manera que todo un complejo filosófico y un círculo de problemas son transformados semánticamente y definidos de nuevo, sobre los que se darán más adelante algunos ejemplos.

\subsection{DUPLICACIÓN DEL SIGNIFICADO}

Es muy frecuente que en Nietzsche una palabra conserve junto a su significado tradicional uno o más significados nuevos adicionales. Politycki formula este hecho de la siguiente manera: «casi cada palabra gana al menos [en el uso del lenguaje de Nietzsche] un doble suelo», así por ejemplo una expresiones centrales como 'ciencia', 'voluntad' y 'máscara'. Incluso llega a afirmar que «las denotaciones Über-, es decir 'hombre superior', oscilan de un extremo a otro» ${ }^{16}$. Intentemos en primer lugar aproximarnos paso a paso al hecho indicado.

Se trata, como se podría formular brevemente, de dobles atribuciones semánticas, de palabras que Nietzsche usa tanto con el significado tradicional como también con un significado nuevo atribuido por él. Palabras con una doble atribución las usa Nietzsche en toda su obra, variando con estos dos significados, es decir, con un significado tradicional y uno nuevo, a diferencia de palabras con cambio de significado, en donde el significado antiguo ya no es usado por Nietzsche.

Lo ejemplos más conocidos son: 'bien' (gut), pero también 'virtud' (Tugend) e incluso 'rebaño' (Herde). Así conserva, es decir, mantiene la palabra 'bien' su significado tradicional en el contexto de la moral cristiana, pero recibe adicionalmente un nuevo significado atribuido por Nietzsche dentro del contexto del hombre noble en la antigua Grecia. Como ejemplo se podría citar también la palabra 'ascesis' (Askese), que en conexión con los antiguos indica en Nietzsche la represión de los instintos sobre la base de su afirmación (JGB §229); sin embargo, ella significa, en cuanto ideal cristiano, la negación y la destrucción de los instintos. Además la palabra 'modestia' (Bescheidenheit), que en el contexto cristiano significa una voluntaria falta de autoestima, pero que en Nietzsche indica una autoestima adecuada, comedida. La palabra 'casta' (Kaste) utilizada por Nietzsche raramente, unida tradicionalmente con una actitud de pureza, conserva en él el significado amplio de 'grupo social, estrato, clase'.

${ }^{16}$ Ibid., p. 107. 
Las palabras pueden tener también varios significados, uno tradicional y otro nuevo adicional, como por ejemplo, la palabra 'moral', que puede indicar por un lado la moral cristiana; pero también, en segundo lugar, puede desempeñar como un concepto genérico para todas las posibles morales; y en tercer lugar, puede indicar en Nietzsche un sistema de valoraciones fundado antropológicamente, que coincide con las condiciones de vida de un ser y le garantiza su existencia.

El significado múltiple de la palabra 'verdad' es sumamente complejo en Nietzsche. Martin distingue: tres uses, es decir frames con diversas variantes de significado respectivamente ${ }^{17}$ De este modo, pueden distinguirse: en primer lugar, los usos de 'verdad' en el contexto de una «crítica de la verdad tradicional» (con 5 variantes de significado); en segundo lugar, los «usos específicamente filosóficos conectados a menudo con reflexiones epistemológicas» (con 6 variantes de significado); y tercero, «usos de la palabra conectados con el nihilismo y la posibilidad de su superación» (con 3 variantes), con lo cual se produce para Martin un significado con catorce variantes, 'la verdad dionisiaca': «la visión de un modo de existencia transformada más allá del nihilismo y la muerte de Dios».

¿Qué pasa, entonces, en este contexto con el problema de la oposición, o polaridad? Politycki vuelve hacer aquí una formulación bastante extrema: «casi todos los conceptos centrales de Nietzsche [están] en sí polarizados» ${ }^{18}$. Un poco antes habla en el contexto presente de 'equivocaciones', y de la presencia de significados contrapuestos en un espacio muy reducido ${ }^{19}$. Como ejemplos cita: 'natural' (KSA XII 449, 10[77]) y 'superficial' (KSA XII 46 y 49, 1[161] y 1[172]) Widhammer habla de 'una polarización interna de la palabra' ${ }^{20}$. ¡Esta tesis va seguramente demasiado lejos! Quizás es valida para 'bien' o 'virtud' -aunque aquí los dos significados correspondientes no están en ningún caso en el mismo plano, pues tienen lugar en contextos respectivamente distintos, no son por consiguiente comparables con otros sin más.

Tomemos todavía otro ejemplo, en el que se trata de un caso especial en tanto que aquí entran en juego varios significantes, que se aproximan mucho pero fonéticamente. Se trata de un caso en que una palabra de Nietzsche conserva adicionalmente dos nuevos significados, en donde estos nuevos significados, sin embargo, se expresan por varios significantes fácilmente cambiados. Pensemos en la palabra 'espíritu libre' (Freigeist). Freigeist tiene en Nietzsche también por de pronto el significado tradicional de 'libertino' y conserva luego adicionalmente otros dos nuevos significados: 'espíritu libre/que ha llegado a ser libre' (el que se libra completamente de todas las tradiciones y crea nuevos

${ }^{17}$ G. T. Martin, From Nietzsche to Wittgenstein, P. Lang. Bern, Frankfurt a.M., 1989, aquí pp. 91-103.

${ }^{18}$ Politycki, op. cit., p. 112.

${ }^{19}$ Ibid., p. 108.

${ }^{20} \mathrm{H}$. Widhammer, Realismus und klassizistische Tradition. Zur Theorie der Literatur in Deutschland 1848-1860, Diss. Tübingen, 1972, aquí p. 102 (citado en Politycki, p. 111, nota 273). 
valores) así como 'legislador' (Gezetzgeber). Para los nuevos significados Nietzsche usa también, sin embargo la palabra 'espíritu libre' (por ejemplo MA, prólogo 2, KSA II 15; EH MA 1, KSA VI 322) ${ }^{21}$. En este caso Politycki vuelve a hablar aquí 'contrario' (Gegenteil): un 'espíritu libre' (freier Geist) indica «directamente lo contrario de aquello que se comprende comúnmente bajo un 'un espíritu libre'» (Freigeist) $)^{22}$. Pero se podría tratar aquí en primer lugar de un seguir progresando en una escala, por lo tanto de una gradación: el 'espíritu libre' (frei Geist) liberándose de la tradición va más allá que el 'espíritu libre' (Freigeist), al que Nietzsche ¡también señala como «(todavía) un espíritu dependiente»! Sin embargo, se podría hablar quizás también, sobre la base de esa distinción de grado llevada hasta el extremo, de una oposición entre el 'espíritu libre' (Freigeist) tradicional y el 'espíritu libre' (frei Geist) de Nietzsche, pero no en el sentido de una contradicción, sino en el sentido de Nietzsche de una lucha, de un combate que busca la libertad en el concepto tradicional de 'espíritu libre' (Freigeist).

\subsection{TRASGRESIÓN DE LOS SIGNIFICADOS POLARES MEDIANTE LA CREACIÓN DE UNO NUEVO, TRASGREDIÉNDOLO Y ABARCÁNDOLO}

Las palabras pueden conservar en Nietzsche un nuevo significado en la medida en que ellas son sacadas de una relación polar tradicional, es decir, de una oposición, cuyos conceptos por su lado han conservado previamente nuevos significados. Un ejemplo de ello: la oposición tradicional 'verdad' versus 'error' desaparece en Nietzsche, pues según su concepción no hay una 'verdad' en sentido metafísico y tradicional. Si no hay ninguna 'verdad' en este sentido, tampoco hay un 'error' como su polo contrario. Todo lo que se hace pasar por 'verdad' es según eso (al mismo tiempo) un 'error'. La palabra 'error' conserva por eso en Nietzsche un nuevo significado más allá de la oposición tradicional, 'verdad versus error'. Todo conocimiento es un 'error' en este sentido, puesto que lo produce el hombre. Pero el hombre tiene que conformarse con estos 'errores', pues él no tiene otra(s) 'verdad'(es). 'Verdad' y 'error' se malogran, y la palabra 'error' conserva por eso sobre esta vía en Nietzsche una connotación positiva.

De modo semejante se conserva la oposición tradicional de los dos términos 'ser' versus 'apariencia'. Se resuelve a favor de 'apariencia' con un nuevo significado. Bönig describe este proceso como 'destornillar la bivalencia' ${ }^{23}$. Análogos procedimientos se pueden también dar, cuando la pareja de contrarios

${ }^{21}$ Más complicado es todavía este hecho a causa de que los nuevos significados no pueden ser fijados sobre un eje cronológico lineal.

${ }^{22}$ Politycki, op. cit., p. 105.

${ }^{23}$ T. Böning, Metaphysik, Kunst und Sprache beim frühen Nietzsche, De Gruyter, Berlin, 1988, aquí p. 95. Vése también C. Aydin, Zijn en Worden. Nietzsches Omduiding van het Substantiebegrip, Diss. Nijmegen, 2003, aquí p. 107. 
'mundo verdadero' versus 'mundo real' se resuelve y se supera a favor de un mundo. También un nuevo concepto de Nietzsche de 'amigo' (Freund) podía llevarse a cabo por este camino. En Nietzsche la palabra 'amigo' conserva un nuevo significado, abarca al mismo tiempo los componentes de los significados tradicionales de 'enemigo' (Feind) y 'amigo', sin corresponderse todavía con los significados tradicionales de ambas palabras.

Este modelo de superación no se observa ciertamente con mucha frecuencia en Nietzsche, sin embargo es fundamental, puesto que se consigue un desmantelamiento de las oposiciones tradicionales, de los pares de oposiciones. Todavía no se pude hacer una indicación más exacta del número de estos procedimientos semánticos; serán 'descubiertas' en parte con el trabajo del Diccionario de Nietzsche.

La centralidad de ese modelo indicado, se pone de manifiesto en el desarrollo semántico filosófico de la pareja de conceptos tradicional 'dionisiaco/apolíneo'. A través de un repetido cambio de oposición el concepto de lo 'dionisiaco' en Nietzsche es cada vez más importante, hasta que él finalmente absorbe en sí el par de oposiciones inicial. Este proceso semántico y filosófico se lleva a cabo en varios pasos. En un primer paso lo 'bárbaro' dionisiaco (mencionado sólo una vez en GT) es refrenado por lo apolíneo hasta convertirse en lo 'dionisiaco griego' ${ }^{24}$. Este 'dionisiaco griego', que en GT ocupa una posición central hasta que entra en escena Sócrates y Eurípides, se acerca por segunda vez a lo apolíneo en una oposición, es decir, 'antagonismo', que produce en el punto álgido de la cultura griega la tragedia griega al unirse estos dos 'instintos artísticos' (por un tiempo). En una tercera fase surge por medio de Sócrates una nueva oposición entre lo dionisíaco y lo socrático (GT 12): arte y ciencia se enfrentan y forman la nueva oposición. Sólo cuando Sócrates comienza a cultivar la música, se produce también aquí la posibilidad de un cambio fecundo entre ciencia y $\operatorname{arte}^{25}$. Sólo así puede lo socrático ser incluido en lo dionisiaco.

Como se ve, el concepto, y con ello el significado de lo 'dionisiaco', llega a ser cada vez más 'importante', en la medida en que incluye en sí paso a paso sus oposiciones. En este proceso semántico y filosófico no se trata, sin embargo, de una dialéctica según el modelo de Hegel, como el mismo Nietzsche pone de relieve varias veces con énfasis, sino más bien de un apoyo en la filosofía de la lucha, de las oposiciones y del juego de Heráclito ${ }^{26}$. Pues en el concepto de lo dionisiaco del Nietzsche tardío no se destruyen los antagonistas mencionados, sino que permanecen conservados en una pluralidad llena de tensión. Según esto, lo dionisiaco tendría que entenderse como una pluralidad dinámica en sí llena de tensión de fuerzas que tienden a enfrentarse.

${ }^{24}$ Sobre esto, B. von Reibnitz, Ein Kommentar zu Friedrich Nietzsche, «Die Geburt der Tragödie aus dem Geiste der Musik» (Kap. 1-12), Metzler, Stuttgart, 1992, pp. 96ss.

${ }^{25}$ GT 13s.; B. von Reibnitz, op. cit., pp. 336-339.

${ }^{26}$ T. Böning, op. cit., p. 220. 


\section{Desciframiento y PRODUCción DE SignificAdos EN los teXtos De NiETzSChE}

\subsection{EL 'RECONOCIMIENTO’ DE SIGNIFICADOS EN LOS TEXTOS DE NIETZSCHE}

Si las palabras (significantes) en los textos de Nietzsche pueden tener significados propios, antiguos y nuevos: ¿cómo, y en qué puede reconocer el lector estos significados? En parte mediante las indicaciones que el mismo Nietzsche incorpora en los textos; pero también en parte faltan tales indicaciones explícitas. Incluso se puede decir que en Nietzsche faltan en gran parte pasajes metalingüísticos, en los que él introduce explícitamente sus nuevos significados o conceptos definidos nuevamente con más detalle. La causa de esto puede quizás estar en que él no quiere fijar significados para siempre.

El reconocimiento de los significados está esencialmente indicado en los siguientes factores. En primer lugar proporciona comprensiblemente el contexto correspondiente una llave a la investigación semántica de las palabras usadas en ellos. El contexto específico de los significados verbales es una nota típica del uso lingüístico de Nietzsche, por medio del cual se distingue en gran medida de la tradición filosófica. Como ejemplo podemos remitirnos al título de GM I: 'bueno y malvado' (Gut und Böse), ‘bueno y malo' (Gut und Schlecht) (GM I, KSA V 257). Aquí las polaridades citadas correspondientes a 'bueno', por consiguiente el contexto, se refieren a los significados distintos de 'bueno', y al mismo tiempo son perfilados también a través de ello los significados de 'malvado' y 'malo' en este título.

También las comillas pueden tener en Nietzsche, junto a otras funciones, una función que modifica el significado y lo denota. Ellas pueden indicar un nuevo significado añadido por Nietzsche; o modificar un nuevo concepto o incluso ironizar, sin que se siga el añadido (explícito) de un nuevo significado. Para eso se puede de nuevo remitir al ejemplo citado más arriba: falta el 'hombre culto'.

Según Stegmaier ${ }^{27}$ Nietzsche pone casi todos los conceptos tradicionales entre comillas, con lo que estas, según su concepción, tienen las siguientes funciones: sirven para poner en duda la pretensión de firmeza de ellos o para poner en duda su base metafísica (como cuando Nietzsche pone entre comillas 'voluntad libre (freier Wille); o al planteamiento de su 'sentido', que Nietzsche busca aclarar entonces por medio de su método genealógico, así por ejemplo el ‘sentido’ de ‘castigo’ (Strafe) (en GM II §12 y §13). Las comillas juegan en Nietzsche un gran papel, de tal manera que él mismo califica su filosofía, aunque también con reservas, de 'filosofía palmípeda' (KSA XI 580, 37[5]) ${ }^{28}$.

El cambio de significado y su especificación puede también efectuarse por medio de la adición de adjetivos determinados como 'pequeño/grande',

\footnotetext{
${ }^{27}$ W. Stegmaier, Nietzsches «Genealogie der Moral», Wissenschaftliche Buchgesellschaft, Darmstadt, 1994, p. 78; reproducido aquí en forma abreviada.

${ }^{28}$ Para esto también U. Willers, op. cit., p. 37.
} 
'verdadero/falso', 'fuerte/débil' y ser reconocido en eso. Según Politycji ${ }^{29}$, 'el adjetivo calificativo 'grande' (gross) puede cambiar el significado de un sustantivo hasta en lo contrario. Como ejemplo citemos, sin que tengamos que entrar aquí una vez más en la cuestión, en qué medida aquí puede hablarse de 'contrario': 'el gran criminal' (KSA XIII 109, 11[283]); 'la gran salud' (FW $\$ 382$, KSA III 653s.); 'la gran razón’ (Z I, KSA IV 39), con lo que se quiere decir la razón del cuerpo, por eso la razón tradicional se entiende también como una 'pequeña razón' ${ }^{30}$.

Politycki distingue aquí también incluso cuatro grados del cambio de significado añadiendo el adjetivo 'grande' ${ }^{31}$. El adjetivo añadido 'grande' contiene siempre una revalorización del sustantivo respectivo, mientras 'pequeño' (klein) consigue lo contrario. Para la asignación de 'grande', o bien 'pequeño', es decisivo si el concepto cuestionado se asocia a la vida fuerte 'ascendente' o a la vida débil 'descendente', o sea a la voluntad de vivir. En este sentido, también es de nuevo palpable, que no se pueden separar muy a menudo en Nietzsche los procesos semánticos y los connotativos, lo que aquí sin embargo fue hecho por razones de claridad.

Los cambios de significado pueden también efectuarse por medio de una especificación del papel del hablante, así cuando por ejemplo se dice: «dicho teológicamente-préstese atención, pues raras veces hablo yo como teólogo...» (EH X §2, KSA VI 351), También aquí se podrían aducir otros ejemplos.

\section{2. Ó PTICAS}

Con los últimos ejemplos fue introducido un hecho, que en los textos de Nietzsche juega un papel muy importante para la atribución -y también nueva atribución- de significados. Nietzsche mismo habla aquí de 'óptica(s)' y también de 'perspectivas' ${ }^{32}$. Con ello se piensa en el hecho de que Nietzsche en sus textos no habla desde un único punto de vista, no desde un única 'óptica', es decir, 'perspectiva'. Más bien, las ópticas pueden cambiar en un mismo texto, y la óptica que habla actualmente en el texto no debe ser su propia óptica. Ópticas particulares, como por ejemplo la cristiana, están acompañadas respectivamente de un uso del lenguaje específico para ellas. Por eso se pude decir, que en los textos de Nietzsche eventualmente las variedades semánticas entran una con otra en una contienda intencionada o también en diálogo. Nietzsche podría querer hacer referencia con ello por un lado a la polisemia de muchas

\footnotetext{
${ }^{29}$ Politycki, op. cit., p. 113.

${ }^{30}$ Los ejemplos se pueden multiplicar fácilmente: el 'gran estilo', la 'gran seriedad' 'la gran misión', etc.

${ }^{31}$ Politycki, op. cit., p. $114 \mathrm{~s}$.

${ }^{32}$ Sobre esto véase C. Zittel, 'Perspektivismus', en H. Ottmann (ed.), Nietzsche Handbuch, Metzler, Stuttgart, 2000, pp. 299-301. T. Busch, Die Affirmation des Chaos. Eos Verlag Erzabtei St. Ottilien, St. Ottilien, 1989, p. 26 habla de Sichtweisen ('modos de ver').
} 
expresiones, que él mismo a través de la atribución de nuevos significados no raramente con frecuencia también aumenta. Por otro lado, su meta podría ser deshacer significados 'comprobados' y con ello movilizar la semántica de muchas palabras y del lenguaje en general.

Podría ser una empresa inútil querer proponer más o menos una lista completa de todas las ópticas que aparecen en sus textos. La óptica de los griegos, por ejemplo, juega un papel importante, es decir, un discurso y un uso del lenguaje desde la óptica de los griegos, para lo que hay que mencionar aquí ante todo a los presocráticos con Heráclito, cuando se trata de hablar de 'opuestos' y de 'devenir'. Además de eso también juega continuamente un papel importante la óptica de la moral y de la religión cristianas. Hablar desde la óptica de la filosofía de Schopenhauer es algo que se circunscribe, por el contrario, al Nietzsche joven (GT)

Desde las distintas posiciones de estas ópticas se producen en Nietzsche distintas atribuciones de significado en los ámbitos de la 'ontología' (devenir frente a ser) moral (bien/mal, bueno/malvado), virtud, humildad, modestia, egoísmo, etc. Las ópticas pueden alternarse, como se ha dicho, en un texto, como por ejemplo ese es el caso en la metáfora de la serpiente en el Zaratustra. Por un lado es la 'la compañera preferida' de Zaratustra (junto con el león); por otro lado, sin embargo, es también repugnante, como la 'serpiente negra' del nihilismo (Z III, KSA IV 201 s). Una superposición de ópticas está presente a menudo en el uso de las 'comillas'.

\subsection{LA VOLUNTAD DE PODER Y SU FUNCIÓN GENERADORA DE SIGNIFICADO}

Aproximadamente a partir de 1884 Nietzsche desarrolla el concepto de voluntad de poder (Z II, KSA IV 146s.), concepto que se puede caracterizar brevemente con Gerhardt como una 'fuerza fundamental de todo acontecer' ${ }^{33}$. Aquí, sin embargo, solamente debe ser aclarado este concepto en su función generadora de significado.

Nietzsche explicita esta función muy claramente una vez en GM (GM II $\S 12$ y $\$ 13$, KSA V 313s), en donde él señala en el ejemplo de la palabra 'pena' (Strafe) el cambio del 'sentido', es decir, significados de una palabra como «una ininterrumpida cadena indicativa de interpretaciones y reajustes siempre nuevos», actúa a través de «la sucesión de procesos de avasallamiento más o menos profundos [...] que tienen lugar en la cosa» (GM II §12, KSA V 314). Nietzsche quiere mostrar con eso que una palabra y una cosa no tienen desde su origen su significado de una vez por todas (él polemiza aquí con Aristóteles), sino que ellas correspondientemente conservan a causa de las constelaciones de poder cambiantes 'sentidos' distintos sucesivos, es decir, significados, o reciben añadidos. Esto lleva a una licuación del significado(s) de una palabra,

${ }^{33}$ V. Gerhardt, 'Wille zur Mach', en H. Toman (ed.), Nietzsche Handbuch, Metzler, Stuttgart, 2000, pp. 353-354. 
por ejemplo de la palabra 'pena'. No sólo la 'forma' introducida por Aristóteles como fija es 'fluida', sino también el 'sentido', los 'sentidos', es decir, los significados de la palabra que indica esta forma, es decir aquí de la palabra 'pena', son fluidos: «la forma es fluida, el 'sentido' lo es todavía más...» se dice en GM (GM II §12, KSA V 315) ${ }^{34}$. En el caso de la 'pena' Nietzsche distingue entre lo 'relativamente duradero en la pena, el uso', por un lado, y por otro lado 'lo fluido en ella, el sentido, la finalidad' (GM II §13, KSA V 216). Ambos están sujetos a las interpretaciones de la 'voluntad de poder' (VP). El 'uso' fue interpretado como 'pena', y a la 'pena' así concebida le fueron atribuidos de nuevo diferentes 'sentidos' y 'finalidades' por sucesivas interpretaciones en el desarrollo cultural (GM II §13, KSA V 317). La voluntad de poder aparece así como una fuerza que interpreta y crea significado ${ }^{35}$. De lo dicho parece claro que no solo hay un 'cambio de significado' en la historia de tradiciones e instituciones (como en el ejemplo de la 'pena'), sino también en el uso propio de Nietzsche de palabras y conceptos.

Las tipificaciones de los opuestos que aparecen no raras veces en el contexto de la VP indican con ello a menudo solamente grados intermedios. Lo mismo pasa, por ejemplo, con la famosa tipificación de los opuestos 'moral de esclavos y moral de señores', que se orienta en la VP. Se introduce en Más allá del bien y del mal (\$260, KSA V 211) e inmediatamente se vuelve a suavizar, pues Nietzsche alude al mismo tiempo en su introducción a que estas dos morales aparecen siempre mezcladas en las 'culturas superiores' ${ }^{36}$.

Mencionemos todavía otro ejemplo para la atribución de un nuevo significado mediante la VP, la palabra 'felicidad' (Glück). En la tradición se circunscribió más bien 'felicidad' con conceptos como 'tranquilidad, paz, satisfacción'. Nietzsche, por el contrario, concibe 'felicidad' sobre la base de la VP ante todo como una acción continuada, sin meta, y de ninguna manera como 'un valor supremo'. El Nietzsche tardío pone luego en conexión 'felicidad' también con su concepto del 'eterno retorno ', del 'decir sí', de tal manera que aquí quizás surge un significado de 'felicidad' que trasciende a los dos mencionados, es decir al tradicional y al suyo propio definido en VP, con lo que aquí también al mismo tiempo podría ser otro ejemplo para la superación de la 'oposiciones'

${ }^{34}$ Cf. sobre esto de una manera más detallada en C. Aydin, op. cit., pp. 115-121.

${ }^{35}$ W. Stegmaier, op. cit., pp. 118-121 aclara el fenómeno de los 'cambio de sentido' en los cinco significados sucesivos, que conserva la palabra Ressentiment según su interpretación en Nietzsche. También juegan un papel central en estos cambios de sentido los factores Stärke ('fortaleza'), o bien Schwäche ('debilidad').

${ }^{36}$ Sobre el papel de la tipificación de la oposición y de su resolución en el contexto presente cf. también W. Stegmaier, op. cit., pp. 118-121. 


\subsection{TRANSVALORACIÓN(ES)}

De acuerdo con su crítica a las oposiciones, tanto en sentido metafísico como también en sentido lógico, pone Nietzsche a menudo gradaciones, diferencias de grados, estadios nuevos y transiciones fluidas en lugar de los significados existentes, en vez de nuevas oposiciones, es decir, de significados contrapuntos en el sentido indicado. Eso ya fue aclarado más arriba. No obstante, hay que mantener que la lucha es un componente integral, no solo para la concepción de Nietzsche de la realidad, sino también de su proyecto filosófico, muy especialmente en su carácter como 'transvaloración'. Y en el contexto de la transvaloración se pude muy bien hablar de oposiciones y contraposiciones, sin embargo (tampoco aquí) en el sentido metafísico tradicional, sino en el sentido de las confrontaciones polémicas llevadas por Nietzsche hasta el extremo con los conceptos y valores tradicionales. En este contexto es, sin embargo, tanto más importante describir de un modo más exacto las operaciones que Nietzsche aplica al lenguaje y a los conceptos, para evitar los malentendidos metafísicos, lógicos o dialécticos de su proyecto de transvaloración. Una transvaloración comprende manifiestamente la transformación de los valores en sus oposiciones, en el sentido de que expresiones con valor positivo cobran un valor negativo, o bien expresiones con valor negativo cobran un valor positivo. Estas sustituciones de valores son producidas, por lo general, mediante una confrontación crítica con expresiones específicas, que tienen que aceptar para ellas su valor positivo, tratando de atribuir a esas mismas expresiones nuevos significados positivos. ¿Qué es lo que ocurre exactamente con eso en los ámbitos del lenguaje, de los conceptos y de sus determinaciones? ¿Qué operaciones aplica Nietzsche a las palabras y a los conceptos, que él somete a la transvaloración? Sobre estos procedimientos el Diccionario de Nietzsche puede arrojar luz de un extremo a otro de los escritos de Nietzsche con su investigación de los significados, variaciones y cambios de significado de las palabras específicas. La palabra 'grandeza' (Grösse), aunque es una expresión de valor, ofrece especialmente buenos ejemplos de la transvaloración. En ellos se pueden distinguir tres tipos de operaciones, tres diferentes estrategias, de dotar al lenguaje con nuevos significados, que son relevantes como tales para el proyecto de la transvaloración.

Para Nietzsche el concepto de 'grandeza' (Grösse, Gr.) desempeña con frecuencia, en conexión con el uso tradicional, como una atribución de un valor positivo (en contextos normativos). En los textos de Nietzsche, sin embargo, el criterio para la Gr. es sumamente variable y dependiente del contexto, y el significado de la palabra Gr. se aparta con frecuencia de los significados habituales. Incluso se pude hablar de un esfuerzo constante por fecundar, transformar y transvalorar recíprocamente conceptos de Gr. tradicionales (cristianos, idealistas) y los actuales (idealistas, morales o políticos). En donde Nietzsche acepta un conflicto con los conceptos existentes de Gr., al menos pueden distinguirse tres estrategias, o bien operaciones: 
La primera estrategia incluye una nueva interpretación crítica de un concepto establecido de Gr., con la intención de aceptar para éste su valor positivo, que se basa sobre el significado que se le ha atribuido hasta ahora. Entonces se atribuye un nuevo significado(s) (o varios) contrapuesto al significado criticado. En este proceso se mantienen las connotaciones positivas de la palabra y son trasladadas a su(s) nuevo(s) significado(s) contrapuesto(s). En consonancia con los nuevos significados se cambian radicalmente los objetos de referencia (denotata). Con este procedimiento la palabra conserva también su valor positivo y su connotación positiva, pero mantiene un nuevo significado contrapuesto y sus denotata se cambian conforme a la modificación del significado.

Así escribe Nietzsche, por ejemplo, en un fragmento póstumo respecto a la moralista 'falsificación de la historia' (KSA XII 428, 9[157]): «se quiere que la $f e$ sea lo sobresaliente de la grandeza». Luego él da a la palabra Gr. un nuevo significado contrapuesto, que parte del concepto de la incredulidad: «pero la falta de objeciones, el escepticismo, la permisión de poderse deshacer de una creencia, la 'inmoralidad' pertenece a la grandeza». El valor positivo de Gr. (ya) no se vincula ahora con la exhibición de la cualidad moral de la fe, sino con la capacidad de pasar sin esta o sin cualidades morales: con 'inmoralidad'. Con esta manera de proceder se cambian los objetos de referencia de Gr., de acuerdo con el nuevo significado de Gr. «Cesar, Federico el Grande, Napoleón pero también Homero, Aristófanes, Leonardo, Goethe [...]». La intención de esta operación es desmoralizar el concepto Gr. y revalorizar a una serie de personas que son consideradas de manera tradicional como no morales o como de dudosa moralidad.

En la segunda operación conserva la palabra Gr. un nuevo significado, pero no por la sustitución de un concepto existente de Gr. -por ejemplo 'fe' (Glaube) por un concepto (incredulidad) radicalmente contrapuesto, sino más bien por medio de que el concepto existente sea sometido a un nuevo sentido que socava la base de su valoración positiva. También aquí la meta consiste de nuevo en conservar las connotaciones positivas de la palabra Gr., pero dándoles en la nueva interpretación del concepto existente una fundamentación completamente nueva. En esta forma de la transvaloración no se cambian, a diferencia de la primera forma, los objetos de referencia de la palabra; lo que se cambia es la manera en que se comprenden.

Así pues, Nietzsche da, por ejemplo, a la palabra Gr. en el Crepúsculo de los ídolos, en las «Incursiones de un intempestivo», § 44, que lleva el título de 'mi concepto de genio', una nueva significación fisiológico-económica: «El genio -en su obra, en su acción- es necesariamente un derrochador: en darse del todo está su grandeza... El instinto de autoconservación queda en suspenso, por así decirlo; la arrolladora presión de las fuerzas que se desbordan le prohíbe toda salvaguarda y toda previsión de ese tipo». Él está de acuerdo entonces con la concepción popular de que la grandeza del genio está en su heroísmo y en su 
espíritu de sacrificio. Él hace esto pero no así, en la medida en que él rechaza este concepto a favor de un concepto radicalmente contrapuesto (por ejemplo, 'crueldad', Grausamkheit, en vez de espíritu de sacrifico); más bien reduce el concepto al nuevo significado de Gr., como derrochar/derroche. A heroísmo y espíritu de sacrificio se les da otra interpretación en conceptos fisiológico-económicos como una imprevisión del derrochador: «A esto se le llama 'holocausto', se alaba en esto su 'heroísmo', su indiferencia frente a su propio bien, su entrega a una idea, a una causa grande, una patria: todas estas cosas son malentendidos... Él se derrama, se desborda, se gasta, no se economiza». En su corrección de los malentendidos de un fenómeno amoral fisiológico Nietzsche no cambia los objetos de referencia de las palabras Gr. y 'genio'. Sus derrochadores no son personas distintas de los héroes de las fantasías populares. Sin embargo, cambia la interpretación de heroísmo como un derrochar el por qué de su grandeza. $\mathrm{Y}$ esto en un modo que excluye la idea de una decisión moral, que es presupuesta por el concepto moral de Gr. como heroísmo y espíritu de sacrificio. La grandeza del genio está ahora en recursos amorales, fisiológicos, que se deben derrochar, «de manera fatal, irremediable, involuntaria, como involuntario es el desbordamiento de un río sobre sus orillas» (Ibid.). En la medida en que se excluye el concepto de libertad de elección y de voluntad, socavan estas metas el concepto moral de Gr. como heroísmo y espíritu de sacrificio y las bases para su valor positivo. Como en el primer ejemplo, esta operación tiene el efecto de desmoralizar el concepto de Gr. pero como un cambio de interpretación del heroísmo tiene ella también el efecto de proporcionar al concepto existente una nueva presuposición fisiológica, o sea superabundancia, riqueza de fuerza, que debe ser derrochada.

En los ejemplos y operaciones considerados hasta ahora se orientan los nuevos significados atribuidos por Nietzsche a la palabra Gr. hasta un cierto grado en los conceptos y significados existentes de Gr. -o como oposición radical (incredulidad en vez de fe) o como una nueva interpretación socavada (heroísmo como deber-derrochar). En eso contrastan estas dos operaciones con una tercera clase de estrategia de transvaloración, que Nietzsche utiliza para atribuir a la palabra Gr. nuevos significados. En este tercer caso funciona la palabra Gr. además como atribución normativa de un valor positivo. Sin embargo, Nietzsche ya no se orienta ahora en la asignación de nuevos significados en un modo reconocible en los conceptos ya existentes de Gr.. Más bien deduce sus criterios para Gr. de sus discusiones críticas con otros conceptos o valores, por ejemplo, con el concepto cristiano de malvado, o a partir de valores de actualidad, como el de la especialización, y además mediante la oposición radical a conceptos o valores criticados. Esta operación aspira, mediante la referencia a regiones o dominios que no tienen una relación explícita o evidente con el concepto de Gr., a la creación de un concepto completamente nuevo de Gr., lo que se indica de vez en cuando mediante el uso de 'comillas'. 
En Más allá del bien y del mal, §212 se presenta el filósofo como «la conciencia malvada de su tiempo». Su secreto propio era siempre «conocer una nueva grandeza del hombre, un nuevo y no recorrido camino hacia su engrandecimiento». Esta descripción proporciona entonces el criterio de la Gr., que se desarrolla en este aforismo, o sea: como lo intempestivo, como aquello que está en la más radical oposición al gusto del tiempo, a las virtudes y tendencias de la época. En el transcurso del texto se usa este criterio sobre tres fenómenos distintos, que exigen tres propiedades intempestivas que luego tienen que formar el nuevo concepto de Nietzsche de Gr.:

1. «A la vista de un mundo de 'ideas modernas', el cual confinaría a cada uno a un rincón y 'especialidad', un filósofo, en el caso de que hoy pueda haber filósofos, se vería forzado a situar la grandeza del hombre, el concepto 'grandeza', precisamente en su amplitud y multiplicidad, en su totalidad en muchas cosas».

2. «Hoy el gusto de la época y la virtud de la época debilitan y enflaquecen la voluntad, nada está tan en armonía con la época como la debilidad de la voluntad: por tanto, en el ideal del filósofo tienen que formar parte del concepto 'grandeza' justo la fortaleza de la voluntad, justo la dureza y capacidad para adoptar resoluciones largas».

3. «Hoy [...] cuando en Europa es el animal de rebaño el único que recibe y reparte honores [...] yo quiero decir, combatiendo conjuntamente todo lo raro, extraño y privilegiado del hombre superior, de la plenitud de poder y el dominio superiores - que hoy el ser aristócrata, el querer ser para sí, el poder ser distinto, el estar solo y el tener que vivir por sí mismo forman parte del concepto 'grandeza'».

La operación puede ser esquematizada del siguiente modo:

Conforme a la época

1. 'Especialidad'

2. 'Debilidad de la voluntad'

3. 'Animal de rebaño' $\rightarrow$ Intempestiva nuevo concepto de 'grandeza'

$\rightarrow$ amplitud, multiplicidad, totalidad en muchas cosas

$\rightarrow$ fortaleza de la voluntad

$\rightarrow \quad$ el ser aristócrata, el poder ser distinto, etc. (combatiendo todo lo raro, extraño, etc.)

Es evidente que estos análisis en cuanto tales (todavía) no pueden ser adecuados o completos. Para una comprensión adecuada de la transvaloración de Nietzsche de Gr. en los textos arriba expuestos deben estos análisis (solamente) puntuales referidos a la expresión 'grandeza' ponerse en conexión con las innovaciones semánticas y transformaciones de la semántica, dentro de un 
amplio marco filosófico de la problemática de la transvaloración nietzscheana. Así, por ejemplo, la operación descrita en el ejemplo del primer texto se puede relacionar con el cambio de la óptica mencionado ya antes: o sea como cambio de una óptica cristiana (grandeza qua fe) a una óptica aristocrática, o bien antigua (grandeza qua incredulidad, falta de objeciones) dentro del amplio marco de una transvaloración: moral aristocrática en contraposición a una moral cristiana. Con ello son manifiestas las primeras combinaciones trasversales entre los primeros procedimientos descritos de la atribución de significados y la(s) transvaloración (es) en sentido estricto.

Pero también el primer análisis posible orientado verbalmente en el Diccionario de Nietzsche trae a la luz, como se acaba de aclarar, algunas importantes distinciones, como por ejemplo: ¿los significados atribuidos como nuevos a una expresión son referidos explícitamente a significados existentes de la expresión (operaciones 1 y 2), o hacen referencia a otras expresiones y conceptos de campos y contextos que están 'fuera' (operación 3)? ¿En el primer caso los nuevos significados son atribuidos a una expresión dada por medio de una oposición radical con significados existentes (operación 1), o mediante una reinterpretación socavada (operación 2)? ¿Conducen los nuevos significados a un cambio de los objetos de referencia del concepto existente (operación 1), o solo a un cambio de la relación de los objetos de referencia existentes (operación 2), o conducen al descubrimiento de serie completamente nueva de objetos de referencia (operación 3)? Tales preguntas podrían abrir la posibilidad de una comprensión más exacta de la transvaloración nietzscheana, especialmente en textos en los que significados existentes de una palabra dada no solo son sometidos a una de las operaciones mencionadas, sino a una mezcla o combinación de estrategias de transvaloración, lo que a menudo parece ser el caso ${ }^{37}$.

traducción de Luis E. De Santiago

Universidad de Málaga

${ }^{37}$ Los ejemplos arriba citados demuestran una distinción ulterior importante y digna de mención, aunque no se pueda seguir discutiendo aquí. En algunos casos, como por ejemplo en el primer texto, una transvaloración contiene un acto de 'legislación', es decir una sustitución formal o nueva definición de conceptos existentes. En otros casos, como en el tercer texto, las formaciones existentes de un concepto son sometidas a un análisis detallado, de manera que las nuevas atribuciones de significados se fundamentan en una argumentación crítica de los conceptos vigentes. Sobre este punto se compare la diferencia entre Umbegreifung y Umwertung ('transvaloración') en Politycki, op. cit., p. 102s. 